Pacific Journal of Mathematic 


\title{
ON AN EXTENSION OF THE PICARD-VESSIOT THEORY
}

\author{
H. F. KREIMER
}

In previous papers, the author has extended the Galois correspondences between differential Picard-Vessiot extensions and algebraic matrix groups to Picard-Vessiot extensions of a wider class of fields with operators, the so-called $M$-fields. In this paper, $M$-field extensions which generalize extensions by integrals and by exponentials of integrals are studied.

These fields are found to be simple field extensions and their structure in the case that the extension is algebraic is investigated. Under suitable restrictions on the fields of constants, the $M$-Galois groups of these fields are shown to be commutative. Criteria are established for such solution fields to be $P-V$ extensions of $M$-fields of difference and differential type. An extension obtained by a finite sequence of algebraic extensions, extensions by integrals, and extensions by exponentials of integrals, is called a generalized Liouville extension. It is demonstrated that if the connected component of the identity element in the $M$-Galois group of a regular $P-V$ extension is a solvable group, then the $P-V$ extension is a generalized Liouville extension, and if a $P-V$ extension is contained in a generalized Liouville extension then the connected component of the identity element in the $M$-Galois group of the $P-V$ extension is solvable.

1, Terminology and notation are briefly considered in $\S 2$, and a preliminary result on the constants of an algebraic $M$-extension of an $M$-field is obtained. The structure of solution fields analogous to extensions by integrals and criteria for the existence of $P-V$ extensions of this type are determined in $\S 3$, and a similar study of solution fields analogous to extensions by exponentials of integrals is made in $\S 4$. In $\S 5$, generalized Liouville extensions are defined, and solvability of the Galois group of a $P-V$ extension is interpreted in terms of imbedding the extension in a generalized Liouville extension.

2. $M$-rings. The terminology and notaion of this paper are the same as in [6] and [7]. Let $C$ be an associative, commutative coalgebra with identity over a ring $W$, which is freely generated as a $W$-module by a set $M$. If $w \rightarrow \bar{w}$ is a homomorphism of $W$ into a ring $S$, let $C^{s}$ be the $S$-module obtained from the $W$-module $C$ by

Received February 21, 1963, and in revised form March 17, 1964. Part of a dissertation presented for the degree of Doctor of Philosophy in Yale University. 
inverse transfer of the basic ring to $S$. If $\rho$ is a homomorphism of a ring $R$ into the algebra $\left(C^{S}\right)^{*}=\operatorname{Hom}_{S}\left(C^{S}, S\right)$; then for every $m \in M$ there is a mapping $a \rightarrow a^{\mathrm{p}}(\mathrm{m})$ of $R$ into $S$, which will also be denoted by $m$, and the set of these mappings will be called an $M$-system of mappings of $R$ into $S$. Let $m \rightarrow \sum_{n p \in M} z_{m n p} n \otimes p$, where $m \in M$, $z_{m n p} \in W$, and $z_{m n p}=0$ except for a finite number of elements $n$ and $p$ in $M$, be the coproduct mapping of $C$ into $C \otimes_{W} C$; if $a, b \in R$ and $m \in M,(a+b) m=a m+b m$ and $(a b) m=\sum_{n, p \in M} \overline{z_{m n p}}(a n)(b p)$. An $M-$ ring is a ring together with an $M$-system of mappings of the ring into itself. An $M$-ring of difference type is an $M$-ring in which the $M$-system of mappings consists of homomorphisms, and an $M$-ring of differential type is an $M$-ring in which the $M$-system of mappings consists of the identity automorphism and higher derivations of rank one or greater.

An element $c$ of an $M$-ring $R$ is a constant if $(c a)^{\rho}=c \cdot a^{\rho}$ for every $a \in R$. The following are equivalent:

(1) $c$ is a constant of $R$,

(2) $c^{\rho}=c \cdot 1^{\rho}$,

(3) $(c a) m=c(a m)$ for every $a \in R$ and $m \in M$,

(4) $c m=c(1 m)$ for every $m \in M$.

The constants of $R$ form a subring of $R$ which contains the identity element of $R$ and this subring will be denoted by $R_{c}$. Suppose $b, d \in R$ and $d$ is a unit in $R$, then $b d^{-1} \in R_{c}$ if, and only if, $d(b m)=b(d m)$ for every $m \in M$. Consequently, if $R$ is a field, so is $R_{c}$.

(2.1) Lemma. Let $K$ be an $M$-field which is an $M$-extension of an $M$-field $L$. If $K$ is an algebraic extension of $L$, then $K_{c}$ is an. algebraic extension of $L_{c}$.

Proof. Suppose $d \in K_{c}$ and $f(x)=x^{h}+a_{h-1} x^{h-1}+\cdots+a_{1} x+a_{0}$ is the irreducible, monic polonomial over $L$ for which $d$ is a root. If $m \in M, 0=(f(d)) m=(f m)(d)$, where $(f m)(x)=(1 m) x^{h}+\left(a_{h-1} m\right) x^{h-1}+\cdots+$ $\left(a_{1} m\right) x+a_{0} m$. But then $(f m)(x)$ must be a multiple of $f(x)$, thus $(f m)(x)=(1 m) f(x)$ and $a_{\alpha} m=(1 m) a_{\alpha}$ for $0 \leqq \alpha \leqq h-1$. Therefore, $a_{\alpha} \in L_{c}$ for $0 \leqq \alpha \leqq h-1$ and $d$ is algebraic over $L_{c}$.

Let $S^{\prime}(M)$ be the free semi-group with identity generated by the set $M$. Operations by elements of $S^{\prime}(M)$ on an $M$-ring $R$ are defined as follows: the identity element of $S^{\prime}(M)$ operates on $R$ as the identity automorphism of $R$, and any other element of $S^{\prime}(M)$ operates on $R$ as the resultant of the operations on $R$ by its factors. If $h$ is a positive integer, $r_{1}, r_{2}, \cdots, r_{h}$ are $h$ elements of $R$, and $s_{1}, s_{2}, \cdots, s_{h}$ are $h$ elements of $S^{\prime}(M)$; denote by $W\left(r_{1}, r_{2}, \cdots, r_{h} ; s_{1}, s_{2}, \cdots, s_{h}\right)$ the determinant: 


$$
\left|\begin{array}{cccc}
r_{1} s_{1} & r_{1} s_{2} & \cdots & r_{1} s_{h} \\
r_{2} s_{1} & r_{2} s_{2} & \cdots & r_{2} s_{h} \\
\vdots & \vdots & & \vdots \\
r_{h} s_{1} & r_{h} s_{2} & \cdots & r_{h} s_{h}
\end{array}\right|
$$

An $M$-field $K$ which is an $M$-extension of an $M$-field $L$ is a solution field over $L$ if there exists a positive integer $h$ and $h$ elements $k_{1}, k_{2}, \cdots, k_{h}$ of $K$, such that $K=L\left\langle k_{1}, k_{2}, \cdots, k_{h}\right\rangle$ and, for some choice of $h$ elements $t_{1}, t_{2}, \cdots, t_{h}$ in $S^{\prime}(M), W\left(k_{1}, k_{2}, \cdots, k_{h} ; t_{1}, t_{2}, \cdots, t_{h}\right)=$ $W_{0} \neq 0$ while $W_{0}^{-1} W\left(k_{1}, k_{2}, \cdots, k_{h} ; t_{1}, \cdots, t_{\alpha-1}, t_{\alpha+1}, \cdots, t_{h}, t\right) \in L$ for $1 \leqq \alpha \leqq h$ and $t=1$ or $t=t_{\beta} m, m \in M$ and $1 \leqq \beta \leqq h$. The set of elements $k_{1}, k_{2}, \cdots, k_{h}$ is a fundamental set for $K$ over $L$. $K$ is a Picard-Vessiot extension of the $M$-field $L$ if $K$ is a solution field over $L$ and, additionally, $K_{c}=L_{c}$ and $L_{c}$ is an algebraically closed field.

\section{Extensions by integrals.}

(3.1) Theorem. Let $K, L$ and $L_{0}$ be $M$-fields such that $K$ is an $M$-exiension of $L$ and $L$ is an $M$-extension of $L_{0}$, and assume there exists $k \in K$ such that $k m-(1 m) k=a_{m} \in L_{0}$ for every $m \in M$.

( i ) $L\langle k\rangle$ is a solution field over $L$.

(ii) If $K_{c}=L\langle k\rangle_{c}$, then $L\langle k\rangle$ is invariant under $M$-automorphisms of $K$ over $L$; and, if $L\langle k\rangle_{c}=L_{c}$, then the $M$-Galois group of $L\langle k\rangle$ over $L$ is commutative.

(iii) As abstract fields, $L\langle l i\rangle$ is a simple extension of $L$ by adjunction of the element $k$.

(iv) $L\langle k\rangle_{c}=L_{c}$ if, and only if, $L\{k\}_{c}=L_{c}$.

( v) If $k$ is algebraic over $L$ but $k \notin L$ and $L\langle k\rangle_{o}=\left(L_{0}\right)_{c}, L$ is a field of characteristic $p \neq 0$ and $k$ is a root of an irreducible polynomial over $L$ of the form $x^{p^{h}}+c_{h-1} x^{p^{h-1}}+\cdots+c_{1} x^{p}+c_{0} x+b$, where $h$ is a positive integer, $c_{\alpha} \in\left(L_{0}\right)_{c}$ for $0 \leqq \alpha \leqq h-1$, and $b_{m}-$ $(1 m) b \in L_{0}$ for every $m \in M$.

(vi) If $L$ is a field of characteristic zero and $k$ is transcendental over $L$ then $L\langle k\rangle_{c}=L_{c}$ if, and only if, there does not exist $b \in L$ such that $b m-(1 m) b=a_{m}$ for every $m \in M$.

(vii) If $L\langle k\rangle$ is a $P-V$ extension, such an extension is unique.

Proof. (i) If $L\langle k\rangle=L$, then $L\langle k\rangle$ is trivially a solution field over $L$ with fundamental set consisting of 1 . Therefore, assume $k \notin L$. If $a_{m}=0$ for every $m \in M$, then $k \in K_{c}$ and $L\langle k\rangle$ is a solution field over $L$ with fundamental set consisting of $k$. If there exists $n \in M$ such that $a_{n} \neq 0$, then the determinant $\left|\begin{array}{cc}1 & k \\ 1 n & k n\end{array}\right|=a_{n} \neq 0$ while 1 and $k$ are solutions of the equations $x m=a_{m} a_{n}^{-1}(x n)+\left((1 m)-(1 n) a_{m} a_{n}^{-1}\right) x$ 
and $(x n) m=\left(a_{n} m+\sum_{m}\right) a_{n}^{-1}(x n)+\left((1 n) m-(1 n)\left(a_{n} m\right) a_{n}^{-1}-(1 n) a_{n}^{-1} \sum_{m}\right) x$ where $\sum_{m}=\sum_{q, r \in M} z_{m q r}((1 n) q) a_{r}$, for every $m \in M$. It is then readily established that $L\langle k\rangle$ is a solution field over $L$ with fundamental set consisting of 1 and $k$.

(ii) An $M$-isomorphism $\varphi$ of $L\langle k\rangle$ over $L$ into $K$ is completely determined by its action on $k$, and $(k \varphi-k) m=(k m) \varphi-k m=$ $\left(a_{m}+(1 m) k\right) \varphi-a_{m}-(1 m) k=(1 m)(k \varphi-k)$ for every $m \in M$. Therefore $k \varphi-k \in K_{c}$ or $k \varphi=k+c$ for some constant $c$. If $K_{c}=L\langle k\rangle_{c}$, then $L\langle k\rangle$ is invariant under $M$-automorphisms of $K$ over $L$; and, if $L\langle k\rangle_{c}=L_{c}$, then the $M$-Galois group of $L\langle k\rangle$ over $L$ is isomorphic to a subgroup of the additive group of constants of $L$.

(iii) The subring $L[k] \subseteq K$ of polynomials over $L$ in $k$ is an $M$ subring of $K$, and $L\langle k\rangle$ is simply the field of fractions of $L[k]$ in $K$. (See Corollary (4.2) of [6]).

(iv) If $L\langle k\rangle_{c}=L_{c}$ then certainly $L\{k\}_{c}=L_{c}$. If $k$ is algebraic over $L$, then $L\langle k\rangle=L[k]=L\{k\}$ and the converse is true. Let $k$ be transcendental over $L$. An element of $L\langle k\rangle$ may be represented as the ratio of a polynomial $f(k) \in L[k]$ and a monic polynomial $g(k) \in L[k]$. Suppose $f(k) \cdot(g(k))^{-1} \in K_{c}$ and is expressed in lowest terms, i.e., $f(k)$ and $g(k)$ are relatively prime. Then $g(k) \cdot((f(k)) m)=f(k) \cdot((g(k)) m)$ for every $m \in M$; and, were $(g(k)) m \neq(1 m) \cdot g(k)$ for some $m \in M$, then $f(k) \cdot(g(k))^{-1}=((f(k)) m-(1 m) f(k)) \cdot((g(k)) m-(1 m) g(k))^{-1}$. This last is impossible since the degree of $(g(k)) m-(1 m) g(k)$ is less than the degree of $g(k)$. Thus $(g(k)) m=(1 m) g(k)$ and $(f(k)) m=(1 m) f(k)$ for every $m \in M$, consequently $f(k), g(k) \in L\{k\}_{c}$. Therefore, if $L\{k\}_{c}=L_{c}$, then $f(k) \cdot(g(k))^{-1} \in L_{c}$.

(v) Suppose $k$ is algebraic over $L$. If $L[y]$ is the ring of polynomials over $L$ in an indeterminate $y$, determined as an $M$-extension of $L$ by setting $y m=a_{m}+(1 m) y$ for every $m \in M$; there is a canonical $M$-homomorphism $\eta$ of $L[y]$ over $L$ into $K$ such that $y^{\eta}=k$. Let $I$ be the kernel of $\eta$, and let $f(y)$ be the monic polynomial which generates $I$, i.e., the minimal polynomial for $k$ over $L$. Because $I$ is an $M$-ideal, $(f(y)) m$ must be a multiple of $f(y)$ and computation shows that $(f(y)) m=(1 m) f(y)$, for every $m \in M$. Therefore $f(y) \in L[y]_{c}$. Suppose $L\langle k\rangle_{c}=L_{c}$ and $g(y) \in L[y]_{c}$. Then $g(k) \in L\langle k\rangle_{c}=L_{c}$, say $g(k)=c$, and $k$ is a root of $g(y)-c$. Therefore $g(y)-c$ is a multiple of $f(y)$ and, if $g(y)$ has positive degree, it is not less than the degree of $f(y)$. Subsequently assume only that $L[y]_{c}$ contains polynomials of positive degree, and $f(y)$ is such a polynomial of least positive degree. If $d \in L_{c},(y+d) m=(1 m)(y+d)+a_{m}$ and $(f(y+d)) m=(1 m) f(y+d)$ for every $m \in M$. Therefore $f(y+d)$ and $f(y+d)-f(y)$ are elements 
of $L[y]_{c}$. The degree of $f(y+d)-f(y)$ is less than the degree of $f(y)$ and, therefore, cannot be positive. Thus $f(y+d)-f(y)=$ $f(d)-f(0)$; but this identity can be valid only if $f(y)$ is a polynomial of degree not greater than one, or $L$ is a field of characteristic $p \neq 0$ and $f(y)=b+\sum_{\alpha=0}^{h} c_{\alpha} y^{p \alpha}$, where $h$ is a nonnegative integer and $b$ and $c_{\alpha}, 0 \leqq \alpha \leqq h$, are elements of $L$. If $\rho$ is the representation of $L[y]$ in $\left(C^{L[y]}\right)^{*}$ associated with the $M$-system of mappings on $L[y]$, then $y^{\rho}=a+y \cdot 1^{\rho}$ where $a$ is that element of $\left(C^{L[y]}\right)^{*}$ such that $a(m)=$ $a_{m}$ for every $m \in M$. If $L$ is a field of characteristic $p \neq 0$ and $f(y)=$ $b+\sum_{\alpha=0}^{h} c_{\alpha} y^{p \alpha}$, then $f(y) \cdot 1^{\rho}=(f(y))^{\rho}=b^{\rho}+\sum_{\alpha=0}^{h} c_{\alpha}^{\rho} a^{p \alpha}+\sum_{\alpha=0}^{h} y^{p^{\alpha}} \cdot c_{\alpha}^{\rho}$. Therefore $c_{\alpha} \cdot 1^{\rho}=c_{\alpha}^{\rho}$ and $c_{\alpha} \in L_{c}$ for $0 \leqq \alpha \leqq h$ and, if $c_{\alpha} \in\left(L_{0}\right)_{c}$ for $0 \leqq \alpha \leqq h$, then $b m-(1 m) b=-\sum_{\alpha=0}^{h}\left(c_{\alpha}^{\rho} a^{p \alpha}\right)(m) \in L_{0}$ for every $m \in M$. The assertion in (v) is now immediate.

(vi) Suppose $L$ is a field of characteristic zero and $k$ is transcendental over $L$. If $L\langle k\rangle_{c} \neq L_{c}$, then $L\{k\}_{c} \neq L_{c}$ and there is a polynomial over $L$ in $k$ of positive degree which belongs to $L\{k\}_{c}$. Let $f(k)$ be such a polynomial of least degree. By the argument in part (v), the degree of $f(k)$ is one. Then $f(k)$ generates a prime $M$-ideal $I$ in $L\{k\}$ and $L\{k\} / I$ is $M$-isomorphic to $L$. If $b$ is the image of $k+I$ under such an $M$-isomorphism, then $b m-(1 m) b=a_{m}$ for every $m \in M$. Conversely, if there exists $b \in L$ such that $b m-(1 m) b=a_{m}$ for every $m \in M$, then $k-b \in L\{k\}_{c}$ and $L\langle k\rangle_{c} \neq L_{c}$.

(vii) Let $L\langle k\rangle$ be a $P-V$ extension of $L$ and let $L\left\langle k^{\prime}\right\rangle$ be a second $P-V$ extension of $L$ such that $k^{\prime} m-(1 m) k^{\prime}=a_{m}$ for every $m \in M$. If $k$ and $k^{\prime}$ are transcendental over $L$, there is an isomorphism $\varphi$ of $L\langle k\rangle$ over $L$ onto $L\left\langle k^{\prime}\right\rangle$ such that $k^{\varphi}=k^{\prime}$ and $\varphi$ is an $M$-isomorphism. Suppose $k$ is algebraic over $L$ and either $k^{\prime}$ is transcendental over $L$ or algebraic over $L$ but of degree over $L$ not less than the algebraic degree of $k$ over $L$. If $f(x)$ is the monic minimal polynomial for $k$ over $L$, then $f\left(k^{\prime}\right) \in L\left\langle k^{\prime}\right\rangle_{c}=L_{c}$ by the argument in part (v); say $f(k)=d$. Then $k^{\prime}$ is a root of $f(x)-d$ and $k^{\prime}$ is algebraic over $L$ with the same degree over $L$ as $k$. If the degree of $k$ over $L$ is one, then $L\langle k\rangle=L=L\left\langle k^{\prime}\right\rangle$. If the degree of $k$ over $L$ is greater than one, then $L$ is a field of characteristic $p \neq 0$ and $f(x)=x^{p^{h}}+$ $c_{h-1} x^{p h-1}+\cdots+c_{1} x^{p}+c^{0} x+b$ where $h$ is a positive integer and $c_{\alpha} \in L_{c}$ for $0 \leqq \alpha \leqq h-1$. Let $c$ be a root in the algebraically closed field $L_{c}$ of $x^{p^{h}}+c_{h-1} x^{p^{h-1}}+\cdots+c_{1} x^{p}+c_{0} x+d$. Then $f\left(k^{\prime}+c\right)=f\left(k^{\prime}\right)$ $-d=0$ and there is an isomorphism $\varphi$ of $L\langle k\rangle$ over $L$ onto $L\left\langle k^{\prime}\right\rangle$ such that $k^{\varphi}=k^{\prime}+c$. $\varphi$ is an $M$-isomorphism.

(3.2) COROLlaRY. Let $L$ be an $M$-field of characteristic zero such that $L_{c}$ is algebraically closed, and let $a_{m}, m \in M$, be elements 
of $L$. There exists a $P-V$ extension $L\langle k\rangle$ of $L$ such that $k m-$ $(1 m) k=a_{m}$ for every $m \in M$ if, and only if (1) there exists an element $b \in L$ such that $b m-(1 m) b=a_{m}$ for every $m \in M$, in which case $L\langle k\rangle=L$, or (2) if $L[y]$ is the ring of polynomials over $L$ in an indeterminate $y$, determined as an $M$-extension of $L$ by setting $y m=$ $a_{m}+(1 m) y$ for $m \in M$, and $L(y)$ is the field of fractions of $L[y]$, then there is a structure of an M-field on $L(y)$ such that $L(y)$ is an $M$-extension of $L[y]$, in which case $L\langle k\rangle$ and $L(y)$ are $M$-isomorphic.

Proof. If there exists $b \in L$ such that $b m-(1 m) b=a_{m}$ for every $m \in M$, set $k=b$ to obtain a trivial $P-V$ extension of $L$. If there does not exist $b \in L$ such that $b m-(1 m) b=a_{m}$ for every $m \in M$, but there is a structure of an $M$-field on $L(y)$ such that $L(y)$ is an $M$ extension of $L[y]$; then $L(y)_{c}=L_{c}$ by part (vi) of Theorem (3.1) and, setting $k=y, L\langle k\rangle=L(y)$ is a $P-V$ extension of $L$. The converse is immediate from parts (iii) and (v) of Theorem (3.1).

If $L$ is an $M$-field of differential type and of characteristic zero such that $L_{c}$ is algebraically closed, Corollary (3.2) may be applied to. establish the existence of $P-V$ extensions by adjunction of integrals.

(3.3) CoRollary. Let $L$ be an $M$-field of difference type such that $L_{c}$ is algebraically closed, and let $a_{m}, m \in M$, be elements of $L$. There exists a $P-V$ extension $L\langle k\rangle$ of $L$ such that $k m-k=a_{m}$ for every $m \in M$ if, and only if, the characteristic is 0 or the following condition is fulfilled when the characteristic is $p \neq 0$ : that there do not exist a nonnegative integer $h, c_{\alpha} \in L_{c}$ for $0 \leqq \alpha \leqq h$, and $b \in L$, such that $b m-b+\sum_{\alpha=0}^{h_{1}} c_{\alpha}\left(a_{m}\right)^{p \alpha}=d_{m} \in L_{c}$ for every $m \in M$, where $\left\{d_{m} \mid m \in M\right\}$ is a finite set not equal to $\{0\}$.

Proof. Let $L[y]$ and $L(y)$ be as in Corollary (3.2). The $M$-system of mappings on $L[y]$ consists of isomorphisms and these can be extended to $L(y)$, so that $L(y)$ is an $M$-field which is an $M$-extension of $L[y]$. Because of Corollary (3.2), only the case when $L$ is a field of characteristic $p \neq 0$ need be considered. If $L[y]_{c}=L_{c}$, then $L(y)_{c}=L_{c}$ by part (iv) of Theorem (3.1) and, setting $y=k, L\langle k\rangle=L(y)$ is the desired $P-V$ extension of $L$. If there exists an irreducible polynomial in $L[y]_{c}$ of positive degree, this polynomial generates a proper prime $M$-ideal $I$ in $L[y]$. The $M$-field $L[y] / I$ is an algebraic extension of $L$ and $(L[y] / I)_{c}=L_{c}$ by Lemma (1.1), since $L_{c}$ is algebraically closed. Setting $k=y+I, L\langle k\rangle=L[y] / I$ is the desired $P-V$ extension of $L$. Therefore, assume that there exist polynomials of positive degree in $L[y]_{c}$, let $f(y)$ be such a polynomial of least positive degree, but assume $f(y)$ is reducible. Analyzing $f(y)$ as in the proof of part (v) of Theorem (3.1), $f(y)$ must have the form $f(y)=b^{\prime}+\sum_{\alpha=0}^{i} c_{\alpha}^{\prime} y^{p^{\alpha}}$ where 
$i$ is a nonnegative integer and $c_{\alpha}^{\prime} \in L_{c}$ for $0 \leqq \alpha \leqq i$. Let $g(y)$ be an irreducible monic polynomial which divides $f(y)$, and let $\zeta$ be a root of $g(y)$ in a splitting field for $f(y)$ over $L$. The roots of $f(y)$ are the elements $\zeta+e$ where $e$ is a root of $f(y)-b^{\prime}$ and lies in the algebraically closed field $L_{c}$. The roots of $g(y)$ are those elements $\zeta+e$ where $e$ is a root of $g(\zeta+y)$. Let $e$ and $e^{\prime}$ be roots of $g(\zeta+y)$; there is an automorphism of the splitting field of $f(y)$ over $L$ which maps $\zeta$ to $\zeta+e^{\prime}$ and its inverse maps $\zeta+e$ to $\zeta+e-e^{\prime}$. Then $g\left(\zeta+e-e^{\prime}\right)=0$, $e-e^{\prime}$ is again a root of $g(\zeta+y)$, and the roots of $g(\zeta+y)$ form an additive subgroup of $L_{c}$. Therefore $g(\zeta+y)$ must be a $p$-polynomial over $L_{c}$, say $g(\zeta+y)=\sum_{\alpha=0}^{h} c_{\alpha} y^{p \alpha}$ where $h$ is a nonnegative integer and $c_{\alpha} \in L_{c}$ for $0 \leqq \alpha \leqq h$; and $g(y)=g(\zeta+(y-\zeta))$ must have the form $g(y)=b+\sum_{w=0}^{h} c_{\alpha} y^{p^{\alpha}}$. Any irreducible monic polynomial which divides $f(y)$ will have the form $g(y+e)$ where $e$ is a root in $L_{c}$ of $f(y)-b^{\prime}$. If $m \in M ;(f(y)) m=f(y),(g(y)) m=g\left(y+e_{m}\right)=g(y)+d_{m}$, and $b m+\sum_{\alpha=0}^{h} c_{\alpha}\left(a_{m}\right)^{p \alpha}=b+d_{m}$, where $d_{m}=g\left(e_{m}\right)-b \in L_{c}$ and $e_{m}$ is a root of $f(y)-b^{\prime}$. Since $g(y)$ is a proper factor of $f(y), g(y) \notin L[y]_{c}$ and $d_{m} \neq 0$ for some $m \in M$.

Conversely, assume there exist a nonnegative integer $h, c_{\alpha} \in L_{c}$ for $0 \leqq \alpha \leqq h$, and $b \in L$, such that $b m-b+\sum_{\alpha=0}^{h} c_{\alpha}\left(a_{m}\right)^{p \alpha}=d_{m} \in L_{c}$ for every $m \in M$, where $\left\{d_{m} \mid m \in M\right\}$ is a finite set not equal to $\{0\}$. Let $E$ be the additive subgroup of $L_{c}$ generated by $\left\{d_{m} \mid m \in M\right\}$, let $g(y)=$ $b+\sum_{\alpha=0}^{h} c_{\alpha} y^{p \alpha}$, let $\bar{f}(y)=\prod_{e \in H}(y+e)$, and let $f(y)=\bar{f}(g(y)) . \quad \bar{f}(y)$ will be a $p$-polynomial over $L_{c}$, i.e. $\bar{f}(y)$ will be a finite linear combination over $L_{c}$ of monomials $y^{p \beta}, \beta$ a nonnegative integer; $f(y)$ will have the form $f(y)=b^{\prime}+\sum_{\alpha=0}^{i} c_{a}^{\prime} y^{p \alpha}$ where $i$ is a nonnegative integer and $c_{\alpha}^{\prime} \in L_{c}$ for $0 \leqq \alpha \leqq i$; and $f(y) \in L[y]_{c}$. If the desired $P-V$ extension $L\langle k\rangle$ existed, $f(k)$ would be an element of $L\langle k\rangle_{c}=L_{c}$. If $c$ is a root in $L_{c}$ of $f(y)-b^{\prime}+f(k)$, then $f(k+c)=0$ and some factor $g(k+c)+$ $e=0$. But then $0=(g(k+c)+e) m=g(k+c)+e+d_{m}=d_{m}$ for every $m \in M$, contrary to the assumption that $\left\{d_{m} \mid m \in M\right\} \neq\{0\}$.

(3.4) CoRollary. Let $L$ be an $M$-field such that the $M$-system of mappings on $L$ consists of the identity automorphism $m_{0}$ and infinite higher derivations and $L_{c}$ is algebraically closed. If $a_{m}, m \in M$ and $m \neq m_{0}$, are elements of $L$, there exists a $P-V$ extension of differential type $L\langle k\rangle$ of $L$ such that $k m=a_{m}$ for every $m \in M, m \neq m_{0}$.

Proof. Let $a_{m_{0}}=0$, and let $L[y]$ and $L(y)$ be as in Corollary (3.2). The $M$-system of mappings on $L[y]$ consists of the identity automorphism $m_{0}$ and infinite higher derivations, and these can be extended to $L(y)$ so that $L(y)$ is an $M$-field of differential type which is an $M$-extension of $L[y]$. By repetition of the argument in the beginning of the proof of Corollary (3.3), only the case when $L$ is a 
field of characteristic $p \neq 0$ and $L[y]_{c}$ contains polynomials of positive degree need be considered. Let $f(y) \in L[y]_{c}$ be a polynomial of positive degree, and let $g(y)$ be an irreducible factor of $f(y)$, say $f(y)=$ $q(y) \cdot(g(y))^{n \cdot p^{i}}$ where $h$ is a positive integer not divisible by $p, i$ is a nonnegative integer, and $q(y)$ is not divisible by $g(y)$. Let $\left\{D_{1}, D_{2}, D_{3}, \cdots\right\}$ be an infinite higher derivation on $L[y]$ contained in the $M$-system of mappings on $L[y]$. If $D_{0}=m_{0},(g(y)) D_{0}=g(y)$. Let $j$ be a positive integer and assume that $(g(y)) D_{\alpha}$ is a multiple of $g(y)$ for $0 \leqq \alpha<j$. Observe that $(g(y))^{p^{i}} D_{\alpha}=0$ for every positive integer $\alpha$ which is not divisible by $p^{i}$ and $(g(y))^{p^{i}} D_{\alpha \cdot p^{i}}=\left((g(y)) D_{a}\right)^{p i}$ for every nonnegative integer $\alpha$. Then $0=(f(y)) D_{j \cdot p i}$ which is equal to a sum of terms divisible by $(g(y))^{h \cdot p^{i}}$ plus the term $h q(y) \cdot(g(y))^{(h-1) p^{i}} \cdot\left((g(y)) D_{j}\right)^{p^{i}}$, and $(g(y)) D_{j}$ must be divisible by $g(y)$. Consequently $g(y)$ generates a proper prime $M$-ideal $I$ in $L[y], L[y] / I$ is an algebraic extension of $L$ and, setting $k=y+I, L\langle k\rangle=L[y] / I$ is the desired $P-V$ extension of $L$.

4. Extensions by exponentials of integrals.

(4.1) Theorem. Let $K, L$, and $L_{0}$ be $M$-fields such that $K$ is an $M$-extension of $L$ and $L$ is an $M$-extension of $L_{0}$, and assume there exists a nonzero $k \in K$ such that $k m=a_{m} k$, where $a_{m} \in L_{0}$, for every $m \in M$.

(i) $L\langle k\rangle$ is a solution field over $L$.

(ii) If $K_{c}=L\langle k\rangle_{c}$, then $L\langle k\rangle$ is invariant under $M$-automorphisms of $K$ over $L$; and, if $L\langle k\rangle_{c}=L_{c}$, then the $M$-Galois group of $L\langle k\rangle$ over $L$ is commutative.

(iii) As abstract fields, $L\langle k\rangle$ is a simple extension of $L$ by adjunction of the element $k$.

(iv) $L\langle k\rangle_{c}=L_{c}$ if, and only if, $L\{k\}_{c}=L_{c}$.

(v) If $k$ is algebraic over $L$ and $L\langle k\rangle_{c}=L_{c}$, then $k$ is a root of an irreducible polynomial over $L$ of the form $x^{h}+b$, where $h$ is $a$ positive integer, $b \neq 0$ and $(b m) b^{-1} \in L_{0}$ for every $m \in M$.

(vi) If $L\langle k\rangle$ is a $P-V$ extension, such an extension is unique.

Proof. (i) It is easily verified that $L\langle k\rangle$ is a solution field over $L$ with fundamental set consisting of $k$.

(ii) An $M$-isomorphism $\varphi$ of $L\langle k\rangle$ into $K$ is completely determined by its action on $k$, and $k((k \varphi) m)=k((k m) \varphi)=k\left(\left(a_{m} k\right) \varphi\right)=$ $\left(a_{m} k\right) \cdot(k \varphi)=(k \varphi) \cdot(k m)$ for every $m \in M$. Therefore $(k \varphi) k^{-1} \in K_{c}$ or $k \varphi=c k$ for some nonzero constant $c$. If $K_{c}=L\langle k\rangle_{c}$, then $L\langle k\rangle$ is invariant under $M$-automorphisms of $K$ over $L$; and, if $L\langle k\rangle_{c}=L_{c}$, then the $M$-Galois group of $L\langle k\rangle$ over $L$ is isomorphic to a subgroup of the multiplicative group of nonzero constants of $L$. 
(iii) The argument is the same as in part (iii) of Theorem (3.1).

(iv) If $L\langle k\rangle_{c}=L_{c}$ then certainly $L\{k\}_{c}=L_{c}$. If $k$ is algebraic over $L$, then $L\langle k\rangle=L[k]=L\{k\}$ and the converse is true. Let $k$ be transcendental over $L$. An element of $L\langle k\rangle$ may be represented as the ratio of a polynomial $f(k) \in L[k]$ and a nonzero polynomial $g(k) \in L[k]$ with either $f(0)=1$ or $g(0)=1$. Suppose $f(k) \cdot(g(k))^{-1} \in K_{c}$ and is expressed in lowest terms. Then $g(k) \cdot((f(k)) m)=f(k) \cdot((g(k)) m)$ for every $m \in M$; and, were $(g(k)) m \neq(1 m) g(k)$ for some $m \in M$, then $f(k) \cdot(g(k))^{-1}=((f(k)) m-(1 m) f(k)) \cdot((g(k)) m-(1 m) g(k))^{-1}$. This last is impossible, since it follows from the equations $f(0) \cdot((g(0)) m-(1 m) g(0))=$ $g(0) \cdot((f(0)) m-(l m) f(0))=0$ that $(f(k)) m-(1 m) f(k)$ and $(g(k)) m-$ $(1 m) g(k)$ are both divisible by $k$. Thus $(g(k)) m=(1 m) g(k)$ and $(f(k)) m=$ $(1 m) f(k)$ for every $m \in M$, consequently $f(k), g(k) \in L\{k\}_{c}$. Therefore, if $L\{k\}_{c}=L_{c}$ then $f(k) \cdot(g(k))^{-1} \in L_{c}$.

(v) Suppose $k$ is algebraic over $L$. If $L[y]$ is the ring of polynomials over $L$ in an indeterminate $y$, determined as an $M$-extension of $L$ by setting $y m=a_{m} y$ for every $m \in M$; there is a canonical $M$ homomorphism $\eta$ of $L[y]$ over $L$ into $K$ such that $y^{\eta}=k$. Let $I$ be the kernel of $\eta$. Since $k \neq 0, y \notin I$. Let $f(y)$ be a polynomial such that $f(y)$ generates $I$ and $f(0)=1$. Because $I$ is an $M$-ideal, $(f(y)) m$ must be a multiple of $f(y)$ and computation shows that $(f(y)) m=$ (1m) $f(y)$, for every $m \in M$. Therefore $f(y) \in L[y]_{c}$. Suppose $L\langle k\rangle_{c}=L_{c}$ and $g(y) \in L[y]_{c}$. Then $g(k) \in L\langle k\rangle_{c}=L_{c}$, say $g(k)=c$, and $k$ is a root of $g(y)-c$. Therefore $g(y)-c$ is a multiple of $f(y)$ and if $g(y)$ has positive degree, it is not less than the degree of $f(y)$. Subsequently assume only that $L[y]_{c}$ contains polynomials of positive degree, and $f(y)$ is such a polynomial of least positive degree. If $b^{-1} y^{n}$ is the highest term of $f(y)$ and $m \in M$, then the identity $(f(y)) m=(1 m) f(y)$ implies $\left(b^{-1} y^{h}\right) m=(1 m) b^{-1} y^{h}$. Therefore $b^{-1} y^{h}$ and $f(y)-b^{-1} y^{h}$ are elements of $L[y]_{c}$. The degree of $f(y)-b^{-1} y^{h}$ is less than the degree of $f(y)$ and, therefore, cannot be positive. Thus $f(y)-b^{-1} y^{h}=f(0)=$ $c \in L_{c} \quad$ or $f(y)=b^{-1} y^{h}+c$. Since $b^{-1} y^{h} \in L[y]_{c}, b\left(y^{h} m\right)=y^{h}(b m)$ or $(b m) b^{-1}=\left(y^{h} m\right) y^{-h} \in L_{0}$ for every $m \in M$. The assertion in (v) is now immediate.

(vi) Let $L\langle k\rangle$ be a $P-V$ extension of $L$ and let $L\left\langle k^{\prime}\right\rangle$ be a second $P-V$ extension of $L$ such that $k^{\prime} \neq 0$ and $k^{\prime} m=a_{m} k^{\prime}$ for every $m \in M$. If $k$ and $k^{\prime}$ are transcendental over $L$, there is an isomorphism $\varphi$ of $L\langle k\rangle$ over $L$ onto $L\left\langle k^{\prime}\right\rangle$ such that $k^{\varphi}=k^{\prime}$ and $\varphi$ is an $M$-isomorphism. Suppose $k$ is algebraic over $L$ and either $k^{\prime}$ is transcendental over $L$ or algebraic over $L$ but of degree over $L$ not less than the algebraic degree of $k$ over $L$. If $x^{h}+b$ is the minimal polynomial for $k$ over $L$, then $b^{-1}\left(k^{\prime}\right)^{h}+1 \in L\left\langle k^{\prime}\right\rangle_{c}=L_{c}$ by the argument in part (v); say 
$b^{-1}\left(k^{\prime}\right)^{h}+1=d$. Then $k^{\prime}$ is a root of $x^{h}+b(1-d)$ and $d \neq 1$. Let $c$ be a root in the algebraically closed field $L_{c}$ of $x^{h}-(1-d)^{-1}$. Then $\left(c k^{\prime}\right)^{h}+b=0$ and there is an isomorphism $\varphi$ of $L\langle k\rangle$ over $L$ onto $L\left\langle k^{\prime}\right\rangle$ such that $k^{\varphi}=c k^{\prime} . \varphi$ is an $M$-isomorphism.

(4.2) CoRollary. Let $L$ be an $M$-field of difference type such that $L_{c}$ is algebraically closed, and let $a_{m}, m \in M$, be elements of $L$. There exists a $P-V$ extension $L\langle k\rangle$ of $L$ such that $k \neq 0$ and $k m=$ $a_{m} k$ for every $m \in M$, if and only if, $a_{m} \neq 0$ for every $m \in M$ and there do not exist positive integers $h$ and $i$ and a nonzero $b \in L$, such that $b m=c_{m}\left(a_{m}\right)^{h} b$ for every $m \in M$, where $c_{m}$ is an ith root of unity and some $c_{m} \neq 1$.

Proof. If the desired $P-V$ extension $L\langle k\rangle$ exists and $m \in M, m$ is an isomorphism on $L\langle k\rangle$. Since $k \neq 0, k m=a_{m} k \neq 0$ and $a_{m} \neq 0$. Therefore assume $a_{m} \neq 0$ for every $m \in M$. Let $L[y]$ be the ring of polynomials over $L$ in an indeterminate $y$, determined as an $M$-extension of $L$ by setting $y m=a_{m} y$ for every $m \in M$, and let $L(y)$ be the field of fractions of $L[y]$. The $M$-system of mappings on $L[y]$ consists of isomorphisms and these can be extended to $L(y)$, so that $L(y)$ is an $M$-field which is an $M$-extension of $L[y]$. If $L[y]_{c}=L_{c}$, then $L(y)_{o}=L_{c}$ by part (iv) of Theorem (4.1) and, setting $k=y, L\langle k\rangle=$ $L(y)$ is the desired $P-V$ extension of $L$. Suppose $f(y) \neq y$ is an irreducible polynomial in $L[y]_{\text {c }}$ of positive degree. $f(y)$ generates a proper prime $M$-ideal $I$ in $L[y], L[y] / I$ is an algebraic extension of $L$, $y \notin I$ and, setting $k=y+I, L\langle k\rangle=L[y] / I$ is the desired $P-V$ extension of $L$. Consequently, assume that there exist polynomials of positive degree in $L[y]_{c}$, let $f(y)$ be such a polynomial of least positive degree, $f(y)$ may be chosen so that $f(0) \neq 0$, but assume $f(y)$ is reducible. Analyzing $f(y)$ as in the proof of part (v) of Theorem (4.1), $f(y)$ must have the form $\left(b^{\prime}\right)^{-1} y^{i}+c^{\prime}$ where $i$ is a positive integer. If $g(y)$ is an irreducible factor of $f(y)$ such that $g(0)=1$, then $g(y)$ has the form $g(y)=b^{-1} y^{h}+1$ where $h$ is a positive integer, and all other such factors of $f(y)$ have the form $g(d y)$ where $d$ is an $i$ th root of unity in $L_{c}$. If $m \in M ;(f(y)) m=f(y),(g(y)) m=g\left(d_{m} y\right)=$ $c_{m}^{-1} b^{-1} y^{h}+1$ and $b m=c_{m}\left(a_{m}\right)^{h} b$, where $c_{m}=\left(d_{m}\right)^{-h}$ and $d_{m}$ is an $i$ th root of unity. Since $g(y)$ is a proper factor of $f(y), g(y) \notin L[y]_{c}$ and $c_{m} \neq 1$ for some $m \in M$.

Conversely, assume there exist positive integers $h$ and $i$ and a nonzero $b \in L$, such that $b m=c_{m}\left(a_{m}\right)^{h} b$ for every $m \in M$, where $c_{m}$ is an $i$ th root of unity and some $c_{m} \neq 1$. Let $g(y)=b^{-1} y^{h}+1$, and let $f(y)$ be the product of the distinct polynomials $g(d y)$ where $d$ is an $h \cdot i$ th root of unity. $f(y)$ will have the form $\left(b^{\prime}\right)^{-1} y^{h \cdot i}+1$ and $f(y) \in L[y]_{c}$. If the desired $P-V$ extension $L\langle k\rangle$ existed, $f(k) \neq 1$ would be an 
element of $L\langle k\rangle_{c}=L_{c}$. If $c$ is a root in $L_{c}$ of $y^{h \cdot i}-(1-f(k))^{-1}$, then $f(c k)=0$ and some factor $g(c d k)=0$. But then $0=(g(c d k)) m=$ $c_{m}^{-1} b^{-1}(c d k)^{h}+1=1-c_{m}^{-1}$ for every $m \in M$, contrary to the assumption that some $c_{m} \neq 1$.

(4.3) CoRollary. Let $L$ be an $M$-field of differential type and of characteristic zero such that $L_{c}$ is algebraically closed. If $m_{0} \in M$ $i s$ the identity automorphism on $L$ and $a_{m}, m \in M$ and $m \neq m_{0}$, are elements of $L$, there exists a $P-V$ extension of differential type $L\langle k\rangle$ of $L$ such that $k \neq 0$ and $k m=a_{m} k$ for every $m \in M, m \neq m_{0}$.

Proof. Let $a_{m_{0}}=1$, and let $L[y]$ and $L(y)$ be defined as in the proof of Corollary (4.2). The $M$-system of mappings on $L[y]$ consists of the identity automorphism $m_{0}$ and higher derivations, and these can be extended to $L(y)$ so that $L(y)$ is an $M$-field of differential type which is an $M$-extension of $L[y]$. By repetition of the argument in the beginning of the proof of Corollary (4.2), only the case when $L[y]_{c}$ contains polynomials of positive degree need be considered. Let $f(y) \in L[y]_{c}$ be a polynomial of positive degree, choose $f(y)$ so that $f(0) \neq 0$, and let $g(y)$ be an irreducible factor of $f(y)$, say $f(y)=$ $q(y) \cdot(g(y))^{h}$ where $h$ is a positive integer and $q(y)$ is not divisible by $g(y)$. Let $\left\{D_{\alpha}\right\}$ be a higher derivation on $L[y]$ contained in the $M$ system of mappings on $L[y]$. If $D_{0}=m_{0},(g(y)) D_{0}=g(y)$. Let $i$ be a positive integer not greater than the rank of $\left\{D_{\alpha}\right\}$ and assume that $(g(y)) D_{\alpha}$ is a multiple of $g(y)$ for $0 \leqq \alpha<i$. Then $0=(f(y)) D_{i}$ which is equal to a sum of terms divisible by $(g(y))^{h}$ plus the term $h q(y) \cdot(g(y))^{h-1} \cdot\left((g(y)) D_{i}\right)$, and $(g(y)) D_{i}$ must be divisible by $g(y)$. Consequently $g(y)$ generates a proper prime $M$-ideal $I$ in $L[y], L[y] / I$ is an algebraic extension of $L, y \notin I$ and, setting $k=y+I, L\langle k\rangle=$ $L[y] / I$ is the desired $P-V$ extension of $L$.

(4.4) COROLlary. Let $L$ be an $M$-field, such that the $M$-system of mappings on $L$ consists of the identity automorphism $m_{0}$ and infinite higher derivations and $L_{c}$ is algebraically closed. If $a_{m}$, $m \in M$ and $m \neq m_{0}$, are elements of $L$, there exists a $P-V$ extension of differential type $L\langle k\rangle$ of $L$ such that $k \neq 0$ and $k m=a_{m} k$ for every $m \in M, m \neq m_{0}$.

Proof. Because of Corollary (4.3), only the case where $L$ is a field of characteristic $p \neq 0$ need be considered. Let $a_{m_{0}}=1$, and let $L[y]$ and $L(y)$ be defined as in the proof of Corollary (4.2). The argument is then analogous to the proof of Corollary (3.4). 


\section{Generalized Liouville extensions.}

(5.1) Definition. An $M$-field $K$ which is an $M$-extension of an $M$-field $L$ is a generalized Liouville extension of $L$ if there exists a positive integer $i$ and $i+1$ intermediate $M$-subfields of $K, L=$ $L_{0} \leqq L_{1} \leqq \cdots \leqq L_{i}=K$, such that for each integer $\alpha, 1 \leqq \alpha \leqq i$, there exists $k \in L_{\alpha}$ such that $L_{\alpha}=L_{\alpha_{-1}}\langle k\rangle$ and

(1) $L_{\alpha}$ is an algebraic extension of $L_{\alpha-1}$, or

(2) $k m-(1 m) k=a_{m} \in L_{\alpha-1}$ for every $m \in M$, or

(3) $k m=a_{m} k$ where $a_{m} \in L_{\alpha-1}$ for every $m \in M$.

If $L$ is an $M$-subfield of an $M$-field $K$, let $A_{K}(L)$ denote the $M$ Galois group of $K$ over $L$. If $G$ is a subgroup of $A_{K}(L)$, let $I(G)$ denote the set of all elements of $K$ left fixed by the automorphisms in $G$; $I(G)$ is an $M$-subfield of $K$ and $L \subseteq I(G) \subseteq K$. Suppose $K$ is a solution field over an $M$-field $L$ such that $K_{c}=L_{c}$ and $k_{1}, k_{2}, \cdots, k_{j}$ is a fundamental set for $K$ over $L$. If $\varphi \in A_{K}(L)$, then $k_{\alpha} \varphi=$ $\sum_{\beta=1}^{j} c_{\alpha \beta} k_{\beta}, 1 \leqq \alpha \leqq j$, where $\left(c_{\alpha \beta}\right)_{1 \leqq \alpha, \beta \leqq j}$ is a matrix over $K_{c}=L_{c}$, by Theorem (3.2) of [7]. The structure of $A_{K}(L)$ may be determined analogously to the analysis of the differential Galois group presented in Kaplansky's An Introduction to Differential Algebra ${ }^{3}$. The results needed in the sequel will be summarized here. $A_{K}(L)$ is an algebraic matrix group over $L_{c}$ and the algebraic subgroups of $A_{K}(L)$ are the subgroups $A_{K}\left(L^{\prime}\right)$ where $L^{\prime}$ is an intermediate $M$-subfield of $K, L \subseteq$ $L^{\prime} \leqq K$. If $H$ is the connected component of the identity element of $A_{K}(L)$, then $H$ is an algebraic subgroup of finite index in $A_{K}(L)$. Therefore $H=A_{K}(\bar{L})$ where $\bar{L}=I(H)$ and $\bar{L}$ is a finite dimensional algebraic extension of $I\left(A_{K}(L)\right)$. Moreover, $\bar{L}$ is algebraically closed in $K$. Indeed, if $k \in K$ is algebraic over $\bar{L}$, then $A_{K}(\bar{L}\langle k\rangle)$ is an algebraic subgroup of finite index in $H$ since the left cosets of $H \bmod A_{K}(\bar{L}\langle k\rangle)$ are in one-to-one correspondence with the distinct images of $k$ under the automorphisms in $H$. Because $H$ is connected, $A_{K}(\bar{L}\langle k\rangle)=H$ and $k \in \bar{L}$.

(5.2) Theorem. Let $K$ be a $P-V$ extension of an $M$-field $L$. If the connected component of the identity element in $A_{K}(L)$ is a solvable group, then $K$ is a generalized Liouville extension of $I\left(A_{K}(L)\right)$.

Proof. Let $H$ be the connected component of the identity element in $A_{K}(L)$ and let $\bar{L}=I(H) . \quad \bar{L}$ is a finite dimensional algebraic extension of $I\left(A_{K}(L)\right)$. Since $H$ is a connected, solvable algebraic matrix group over the algebraically closed field $L_{c}$, a fundamental set $k_{1}, k_{2}, \cdots$, $k_{j}$ for $K$ over $L$ may be chosen so that the $M$-automorphisms of $H$ are represented by triangular matrices, say $k_{\alpha} \varphi=\sum_{\beta=\alpha}^{j} c_{\alpha \beta}(\varphi) \cdot k_{\beta}$ for $\varphi \in H$ and $1 \leqq \alpha \leqq j$, where the coefficients $c_{\alpha \beta}(\varphi) \in L_{c}$. If $m \in M$ and 
$\varphi \in H$, then $\left(\left(k_{j} m\right) k_{j}^{-1}\right) \varphi=\left(\left(k_{j} \varphi\right) m\right)\left(k_{j} \varphi\right)^{-1}=\left(\left(c_{j j}(\varphi) \cdot\left(k_{j} m\right)\right)\left(c_{j j}(\varphi) \cdot k_{j}\right)^{-1}=\right.$ $\left(k_{j} m\right) k_{j}^{-1}$ and $\left(k_{j} m\right) k_{j}^{-1} \in \bar{L}$. Thus $k_{j} m=a_{m} k_{j}$ where $a_{m} \in \bar{L}$ for every $m \in M$. If $m \in M$ and $\varphi \in H$, let $k_{\alpha}^{\prime}(m)=\left(k_{\alpha} k_{j}^{-1}\right) m-(1 m) k_{\alpha} k_{j}^{-1}$ and $c_{\alpha \beta}^{\prime}(\varphi)=c_{\alpha \beta}(\varphi) \cdot\left(c_{j j}(\varphi)\right)^{-1}$ for $\alpha \leqq \beta \leqq j-1$ and $1 \leqq \alpha \leqq j-1$; then

$$
\begin{aligned}
\left(k_{\alpha}^{\prime}(m)\right) \varphi & =\left(\left(k_{\alpha} \varphi\right)\left(k_{j} \varphi\right)^{-1}\right) m-(1 m)\left(k_{\alpha} \varphi\right)\left(k_{j} \varphi\right)^{-1} \\
& =\sum_{\beta=\infty}^{j} c_{\alpha \beta}(\varphi) \cdot\left(c_{j j}(\varphi)\right)^{-1}\left(\left(k_{\beta} k_{j}^{-1}\right) m-(1 m) k_{\beta} k_{j}^{-1}\right) \\
& =\sum_{\beta=\infty}^{j-1} c_{\alpha \beta}^{\prime}(\varphi) \cdot k_{\beta}^{\prime}(m) .
\end{aligned}
$$

By Theorem (3.2) of [7], $K$ is finitely generated as an abstract field over $\bar{L} \supseteqq L$; therefore every intermediate subfield is also finitely generated over $\bar{L}$. Consequently, if $L^{\prime}$ is the $M$-subfield of $K$ generated over $\bar{L}$ by the $k_{\alpha}^{\prime}(m), m \in M$ and $1 \leqq \alpha \leqq j-1$, then there are finitely many $m \in M$ such that $L^{\prime}$ is generated as an $M$-field over $\bar{L}$ by the $k_{\alpha}^{\prime}(m)$ for these $m$ and $1 \leqq \alpha \leqq j-1$. By induction on $j$, it may be assumed that $L^{\prime}$ is a generalized Liouville extension of $I\left(A_{K}(L)\right)$. Since $\left(k_{\alpha} k_{j}^{-1}\right) m-(1 m) k_{\alpha} k_{j}^{-1}=k_{\alpha}^{\prime}(m) \in L^{\prime}$ for every $m \in M$ and $1 \leqq \alpha \leqq j-1$ while $k_{j} m=a_{m} k_{j}$ where $a_{m} \in \bar{L} \cong L^{\prime}$ for every $m \in M$, it follows that $K$ is a generalized Liouville extension of $I\left(A_{k}(L)\right)$.

In connection with this theorem, it should be noted that $I\left(A_{K}(L)\right)=$ $L$ if $K$ is a regular field extention of $L$. If $K$ is an $M$-field of differential type, then $I\left(A_{K}(L)\right)=L$ provided only that $K$ is a separable field extension of $L$.

(5.3) Lemma. Let $K^{\prime}, K, L^{\prime}$ and $L$ be $M$-fields such that $K^{\prime}$ is an $M$-extension of $L, K$ and $L^{\prime}$ are $M$-subfields of $K^{\prime}$ and contain $L$, and $K^{\prime}$ is generated by its subfields $K$ and $L^{\prime}$.

(i) If $K$ is a solution field over $L, K^{\prime}$ is a solution field over $L^{\prime}$ and a fundamental set for $K$ over $L$ is a fundamental set for $K^{\prime}$ over $L^{\prime}$.

(ii) If $K$ and $L^{\prime}$ are linearly disjoint over $L$, there is a canonical isomorphism of $A_{K}(L)$ into $A_{K^{\prime}}\left(L^{\prime}\right)$. Moreover, if $K$ is a solution field over $L, K_{c}=L_{c}, K_{c}^{\prime}=L_{c}^{\prime}$, and $A_{K}(L)$ and $A_{K^{\prime}}\left(L^{\prime}\right)$ are represented by matrices with respect to the same fundamental set for $K$ over $L$ and $K^{\prime}$ over $L^{\prime}$; then this canonical isomorphism is the identity map on matrices.

Proof. (i) The verification is immediate from the definition of solution field.

(ii) If $K$ and $L^{\prime}$ are linearly disjoint over $L$, automorphisms of $K$ over $L$ extend uniquely to automorphisms of $K^{\prime}$ over $L^{\prime}$ and $M$ automorphisms of $K$ over $L$ extend to $M$-automorphisms of $K^{\prime}$ over 
$L^{\prime}$, yielding an isomorphism of $A_{k}(L)$ into $A_{K^{\prime}}\left(L^{\prime}\right)$. The remaining assertion is immediate.

The converse of theorem (5.2) is a consequence of

(5.4) THEOREM. If $K^{\prime}$ is a generalized Liouville extension of an $M$-field $L$ and $K$ is an intermediate $M$-subfield of $K^{\prime}$ such that $K$ is a P-V extension of $L$, then the connected component of the identity element in $A_{K}(L)$ is a solvable group.

Proof. By Corollary (2.3) of [7], $K$ and $K_{c}^{\prime}$ are linearly disjoint over $K_{c}=L_{c}$, whence $K$ and $L\left(K_{c}^{\prime}\right)$ are linearly disjoint over $L$. By Lemma (5.3), $K\left(K_{c}^{\prime}\right)$ is a solution field over $L\left(K_{c}^{\prime}\right)$; and there is a matrix representation for the algebraic group $A_{K}(L)$ over $L_{c}$, a matrix representation for the algebraic group $A_{K\left(K_{c}^{\prime}\right)}\left(L\left(K_{c}^{\prime}\right)\right)$ over $K_{c}^{\prime} \supseteqq L_{c}$, and a canonical isomorphism of $A_{K}(L)$ into $A_{K\left(K_{c}^{\prime}\right)}\left(L\left(K_{c}^{\prime}\right)\right)$ which is the identity map on matrices. If $H$ is the connected component of the identity element in $A_{K}(L)$, then $H$ is an irreducible component of $A_{K}(L)$ and its image in $A_{K\left(K_{c}^{\prime}\right)}\left(L\left(K_{c}^{\prime}\right)\right)$ is irreducible, hence connected, since $L_{c}$ is algebraically closed. Therefore $H$ is mapped into the connected component of the identity element in $A_{K^{\left(K_{c}^{\prime}\right)}}\left(L\left(K_{c}^{\prime}\right)\right)$, and it will suffice to prove the theorem under the assumptions that $K$ is merely a solution field over $L$ but $K_{c}^{\prime}=L_{c}$.

Let $L=L_{0} \subseteq L_{1} \subseteq \cdots \subseteq L_{i}=K^{\prime}$ be as in definition (5.1), and let $k \in L_{1}$ be such that $L_{1}=L\langle k\rangle$ and

(1) $L_{1}$ is an algebraic extension of $L$, or

(2) $k m-(1 m) k=a_{m} \in L$ for every $m \in M$, or

(3) $k m=a_{m} k$ where $a_{m} \in L$, for every $m \in M$.

Be induction on $i$, it may be assumed that the connected component of the identity element in $A_{K\langle k\rangle}\left(L_{1}\right)$ is solvable. Let $\bar{L}=I(H)$, where again $H$ denotes the connected component of the identity element in $A_{K}(L) . \quad K$ is a regular extension of $\bar{L}$, since $\bar{L}$ is algebraically closed in $K$ and $\bar{L}$ is the fixed field of a group of automorphisms of $K$ whence $K$ is a separable extension of $\bar{L}$. If $L_{1}$ is an algebraic extension of $L$, then $\bar{L}\langle k\rangle$ is an algebraic extension of $\bar{L}$ and $K$ and $\bar{L}\langle k\rangle$ are linearly disjoint over $\bar{L}$. The canonical isomorphism of $H=A_{K}(\bar{L})$ into $A_{K\langle k\rangle}(\bar{L}\langle k\rangle)$ given by lemma (5.3) must map $H$ into the connected component of the identity element in $A_{K\langle k\rangle}\left(L_{1}\right)$, whence $H$ is solvable.

Assume $L_{1}$ is not an algebraic extension of $L$. If $k$ is transcendental over $K$, then $L_{1}=L(k)$ and $K\langle k\rangle=K(k)$ by Theorems (3.1) and (4.1). $K$ and $L_{1}$ are linearly disjoint over $L$, so again there is a canonical isomorphism of $H$ into the connected component of the identity element in $A_{K\langle k\rangle}\left(L_{1}\right)$ and $H$ is solvable. Suppose $k$ is algebraic over $K$. If $k m=a_{m} k$ where $a_{m} \in L$ for every $m \in M$, then $k^{h}+b=0$ where $h$ is a positive integer, $b \in K$ and again $(b m) b^{-1} \in L$ for every 
$m \in M$. If $k m-(1 m) k=a_{m} \in L$ for every $m \in M$, then $L$ is a field of characteristic $p \neq 0$ and $k^{p h}+c_{h-1} k^{p h-1}+\cdots+c_{1} k^{p}+c_{0} k+b=0$ where $h$ is a positive integer, $c_{\alpha} \in K_{c}=L_{c}$ for $0 \leqq \alpha \leqq h-1, b \in K$ and again $b m-(1 m) b \in L$ for every $m \in M$. In either case $L\langle b\rangle$ is invariant under the automorphisms in $A_{K}(L)$ and $A_{L\langle b\rangle}(L)$ is commutative, by Theorems (3.1) and (4.1). Therefore, $A_{K}(L\langle b\rangle)$ is an invariant subgroup of $A_{K}(L)$ and the factor group, which is isomorphic to a subgroup of $A_{L\langle b\rangle}(L)$, is commutative. $L_{1}$ is an algebraic extension of $L\langle b\rangle$ and, by a preceding argument, the connected component of the identity element in $A_{K}(L\langle b\rangle)$ is canonically isomorphic to a subgroup of the connected component of the identity element in $A_{K\langle k\rangle}\left(L_{1}\right)$ and is solvable. Therefore $H$, the connected component of the identity element in $A_{K}(L)$, is solvable by Lemma (4.9) of [3].

\section{REFERENCES}

1. R. M. Cohn, Extensions of difference fields, Amer. J. Math. 74 (1952), 507-530.

2. C. H. Franke, Picard-Vessiot theory of linear homogeneous difference equations, Trans. Amer. Math. Soc. 108 (1963), 491-515.

3. Irving Kaplansky, An introduction to differential algebra, Hermann, Paris, 1957. 4. E. R. Kolchin, Algebraic matric groups and the Picard-Vessiot theory of homogeneous linear ordinary differential equations, Annals of Math. 49 (1948), 1-42.

5. — Picard-Vessiot theory of partial differential fields, Proc. Amer. Math. Soc. 3 (1952), 596-603.

6. H. F. Kreimer, The foundations for an extension of differential algebra, Trans. Amer. Math. Soc., III (1964), 482-492.

7. —, An extension of differential Galois theory, accepted for publication by Trans. Amer. Math. Soc.

8. Lawrence Markus, Group theory and differential equations, Lecture notes at the University of Minnesota, Institute of Technology, Department of Mathematics. 



\section{PACIFIC JOURNAL OF MATHEMATICS}

\section{EDITORS}

H. Samelson

Stanford University

Stanford, California

R. M. Blumenthal

University of Washington

Seattle, Washington 98105
J. Dugundu

University of Southern California Los Angeles, California 90007

*Richard Arens

University of California

Los Angeles, California 90024

\section{ASSOCIATE EDITORS}
E. F. BECKENBACH
B. H. NeumanN
F. WOLF
K. YOSIDA

\section{SUPPORTING INSTITUTIONS}

UNIVERSITY OF BRITISH COLUMBIA CALIFORNIA INSTITUTE OF TECHNOLOGY UNIVERSITY OF CALIFORNIA MONTANA STATE UNIVERSITY

UNIVERSITY OF NEVADA

NEW MEXICO STATE UNIVERSITY

OREGON STATE UNIVERSITY

UNIVERSITY OF OREGON

OSAKA UNIVERSITY

UNIVERSITY OF SOUTHERN CALIFORNIA
STANFORD UNIVERSITY

UNIVERSITY OF TOKYO

UNIVERSITY OF UTAH

WASHINGTON STATE UNIVERSITY

UNIVERSITY OF WASHINGTON

AMERICAN MATHEMATICAL SOCIETY CALIFORNIA RESEARCH CORPORATION SPACE TECHNOLOGY LABORATORIES NAVAL ORDNANCE TEST STATION 


\section{Pacific Journal of Mathematics}

\section{Vol. 15, No. $1 \quad$ September, 1965}

Donald Charles Benson, Unimodular solutions of infinite systems of linear

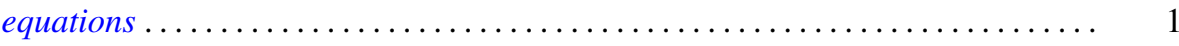

Richard Earl Block, Transitive groups of collineations on certain designs . . . . . . 13

Barry William Boehm, Existence of best rational Tchebycheff approximations .... . 19

Joseph Patrick Brannen, A note on Hausdorff's summation methods . . . . . . . . . . 29

Dennison Robert Brown, Topological semilattices on the two-cell ............ 35

Peter Southcott Bullen, Some inequalities for symmetric means . . . . . . . . . . 47

David Geoffrey Cantor, On arithmetic properties of coefficients of rational

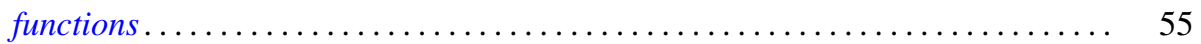

Luther Elic Claborn, Dedekind domains and rings of quotients . . . . . . . . . 59

Allan Clark, Homotopy commutativity and the Moore spectral sequence ........ 65

Allen Devinatz, The asymptotic nature of the solutions of certain linear systems of

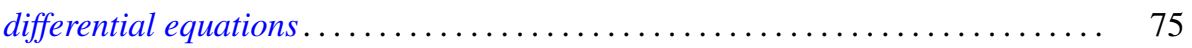

Robert E. Edwards, Approximation by convolutions ................... 85

Theodore William Gamelin, Decomposition theorems for Fredholm operators . . . . . 97

Edmond E. Granirer, On the invariant mean on topological semigroups and on

topological groups .................................. 107

Noel Justin Hicks, Closed vector fields . . . . . . . . . . . . . . . . . . . 141

Charles Ray Hobby and Ronald Pyke, Doubly stochastic operators obtained from

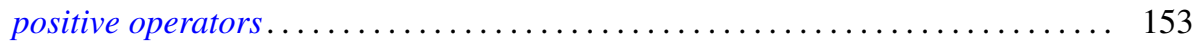

Robert Franklin Jolly, Concerning periodic subadditive functions . . . . . . . . . 159

Tosio Kato, Wave operators and unitary equivalence . . . . . . . . . . . . . . 171

Paul Katz and Ernst Gabor Straus, Infinite sums in algebraic structures . . . . . . . 181

Herbert Frederick Kreimer, Jr., On an extension of the Picard-Vessiot theory ...... 191

Radha Govinda Laha and Eugene Lukacs, On a linear form whose distribution is

identical with that of a monomial ......................... 207

Donald A. Ludwig, Singularities of superpositions of distributions . . . . . . . . . 215

Albert W. Marshall and Ingram Olkin, Norms and inequalities for condition

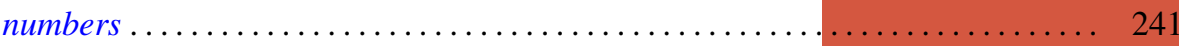

Horace Yomishi Mochizuki, Finitistic global dimension for rings . . . . . . . . . . 249

Robert Harvey Oehmke and Reuben Sandler, The collineation groups of division

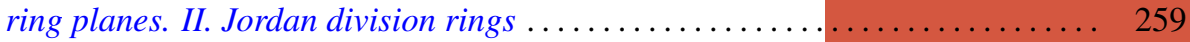

George H. Orland, On non-convex polyhedral surfaces in $E^{3} \ldots \ldots \ldots \ldots \ldots \ldots \ldots 267$

Theodore G. Ostrom, Collineation groups of semi-translation planes . . . . . . . . 273

Arthur Argyle Sagle, On anti-commutative algebras and general Lie triple

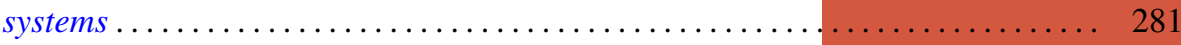

Laurent Siebenmann, A characterization of free projective planes . . . . . . . . . 293

Edward Silverman, Simple areas.................................. 299

James McLean Sloss, Chebyshev approximation to zero .................. 305

Robert S. Strichartz, Isometric isomorphisms of measure algebras . . . . . . . . . 315

Richard Joseph Turyn, Character sums and difference sets . . . . . . . . . . . . 319

L. E. Ward, Concerning Koch's theorem on the existence of arcs . . . . . . . . . . 347

Israel Zuckerman, A new measure of a partial differential field extension ......... 357 